\title{
Cut-wire pairs and plate pairs as magnetic atoms for optical metamaterials
}

\author{
G. Dolling, C. Enkrich, and M. Wegener \\ Institut für Angewandte Physik, Universität Karlsruhe (TH), Wolfgang-Gaede-Strasse 1, \\ D-76131 Karlsruhe, Germany
}

J. F. Zhou and C. M. Soukoulis*

Ames Laboratory and Department of Physics and Astronomy, Iowa State University, Ames, Iowa 50011

\section{S. Linden}

Institut für Nanotechnologie, Forschungszentrum Karlsruhe in der Helmholtz-Gemeinschaft, Postfach 3640, D-76021 Karlsruhe, Germany

\begin{abstract}
Received June 9, 2005; revised manuscript received August 11, 2005; accepted August 16, 2005
We study the optical properties of metamaterials made from cut-wire pairs or plate pairs. We obtain a more pronounced optical response for arrays of plate pairs, a geometry that also eliminates the undesirable polarization anisotropy of the cut-wire pairs. The measured optical spectra agree with simulations, revealing negative magnetic permeability in the range of telecommunications wavelengths. Thus nanoscopic plate pairs might serve as an alternative to the established split-ring resonator design. (C) 2005 Optical Society of America

OCIS codes: $160.4760,260.5740$.
\end{abstract}

Magnetic and left-handed metamaterials have recently attracted considerable attention ${ }^{1-7}$ because of their unusual optical properties ${ }^{8}$ and because of potential applications such as so-called perfect lenses. ${ }^{9}$ These lenses require a negative refractive index, which can be produced by a negative electric permittivity and a negative magnetic permeability at the same frequency. Traditionally, ${ }^{10}$ these properties are achieved by use of a combination of artificial "electric atoms" and artificial "magnetic atoms," i.e., split-ring resonators and metallic wires, with lattice constants much smaller than the wavelength of light, such that the light field experiences an effective homogeneous medium.

Other theoretical work ${ }^{11-13}$ has shown, however, that pairs of finite-length wires (cut-wire pairs) would not only be able to replace the split-ring resonators (SRRs) but possibly could also lead to a negative refractive index directly, without the need for additional metallic wires. It is our aim in this Letter to investigate the optical properties of metamaterials made from such cut-wire pairs and from related plate pairs experimentally and to systematically study their dependence on different design parameters.

Figure 1(a) illustrates the connection between normal SRRs and cut-wire pairs. At the left a normal SRR is shown, which can be viewed as just one winding of a magnetic coil with inductance $L$ in series with capacitance $C{ }^{4}$, which is formed by the ends of the wire. The oscillating magnetic field perpendicular to the SRR plane induces a circulating current in the coil. Near the $L C$ resonance at angular frequency $\omega_{L C}=1 / \sqrt{L C}$, the circulating current in the coil can lead to a magnetic moment perpendicular to the plane of the coil that can counteract the external magnetic field, enabling a negative magnetic permeability to occur. Opening the split in the SRR de- creases capacitance $C$ and hence increases the $L C$ resonance frequency. Additionally, opening the bottom arm of the resultant $U$ shape leads to a second serial capacitance, further reducing the net capacitance in the circuit; this results in an increase of the resonance frequency. Further opening of the lower slit brings us to a pair of cut wires. The result of this transition is that the ohmic currents in the horizontal arms at the left in Fig. 1(a) have been replaced by displacement currents at the right. On the one hand, we have increased the $L C$ resonance frequency for a given minimum feature size. On the other hand, this increased resonance frequency at a fixed lattice constant decreases the ratio between (resonance) wavelength $\lambda$ and lattice constant $a$. A true metamaterial requires a very large ratio of wavelength to lattice constant, typically of the order of $\lambda / a=10$, for a SRR. ${ }^{14,15}$ With the cut-wire-pair design, this ratio is typically $\lambda / a \approx 2$.

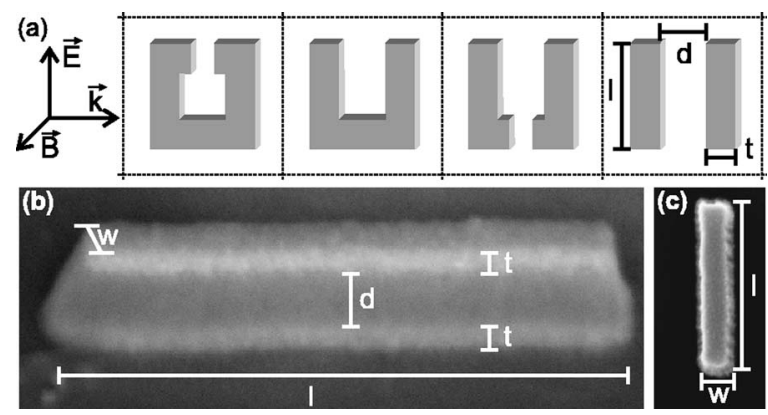

Fig. 1. (a) Schematic of the adiabatic transition from splitring resonators (left) to cut-wire pairs (right) as "magnetic atoms" of optical metamaterials. (b) Electron micrograph (oblique-incidence view) of an actual cut-wire pair with $w$ $=150 \mathrm{~nm}, t=20 \mathrm{~nm}, d=60 \mathrm{~nm}$, and $l=700 \mathrm{~nm}$; (c) corresponding top view. 

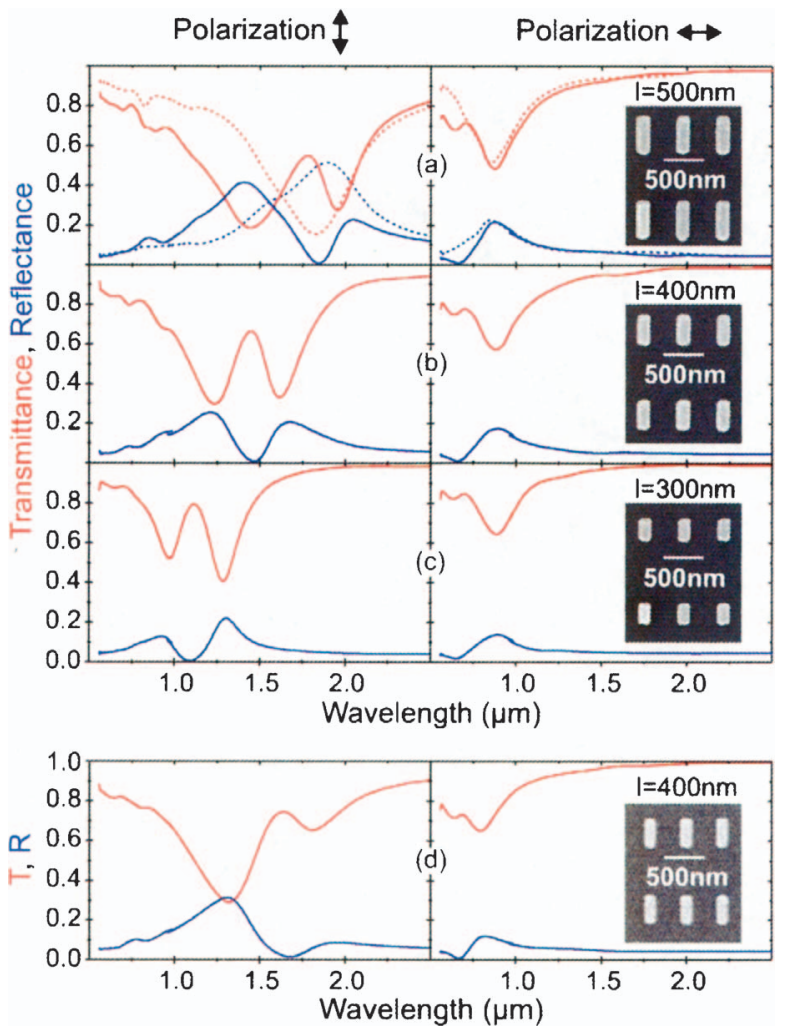

Fig. 2. Measured transmittance (red) and reflectance (blue) spectra for cut-wire pairs for vertical incident polarization (left column) and horizontal polarization (right column). $l=$ (a) 500 , (b) 400, (c) $300 \mathrm{~nm}$. Fixed parameters for (a) $-(\mathrm{c}): w=150 \mathrm{~nm}, t=20 \mathrm{~nm}, d=80 \mathrm{~nm}, a_{x}=500 \mathrm{~nm}$, and $a_{y}=1050 \mathrm{~nm}$. Dotted curves in (a), spectra from a nominally identical structure but without the topmost gold wire. (d) same as (b) but $d=60 \mathrm{~nm}$ rather than $d=80 \mathrm{~nm}$. Insets, the corresponding electron micrographs (top views).

Obvious relevant design parameters are the length of the cut wires $l$, the metal thickness $t$, the width $w$ of the wires, and the spacing between the wires $d$. To fabricate structures suitable for the polarization configuration depicted in Fig. 1(a) on a substrate for normal incidence conditions, one cut wire has to be on top of the other, with a certain dielectric spacer layer between [Fig. 1(b)]. It is straightforward to achieve such a design: We start by defining the lateral structure in a poly(methyl methacrylate) photoresist (spin coated onto a glass substrate covered with a $5 \mathrm{~nm}$ layer of indium tin oxide) by standard electron-beam lithography. Thereafter we sequentially deposit a $t$ $=20 \mathrm{~nm}$ thin gold layer, a $\mathrm{MgF}_{2}$ layer of thickness $d$ and refractive index 1.39, and another $20 \mathrm{~nm}$ thin gold layer, all by electron-beam evaporation, followed by a lift-off procedure. All samples described in this Letter have a total area of $80 \mu \mathrm{m} \times 80 \mu \mathrm{m}$.

Transmittance and reflectance spectra are measured with a Fourier-transform infrared spectrometer (Bruker Equinox 55, NIR halogen source) combined with an infrared microscope (Bruker Hyperion 1000; 36× Cassegrain lens; N.A., 0.5; Si detector or liquid- $\mathrm{N}_{2}$-cooled InSb detector; infrared polarizer). The samples are aligned with their surfaces perpendicular to the optical axis. The transmittance and reflectance spectra are normalized to the bare sub- strate and a silver mirror, respectively, and are taken for two orthogonal linear polarizations of the incident light.

Figures 2(a)-2(c) show results for cut-wire pairs of different lengths but fixed $\mathrm{MgF}_{2}$ spacer thickness and fixed wire width. The dotted curves in Fig. 2(a) correspond to a sample with nominally identical parameters, however, without the topmost gold layer. This single cut-wire sample shows only one pronounced resonance, the Mie resonance, for each polarization. For an incident polarization along the long axis of the cut-wire pairs, two resonances are observed, which essentially disappear for orthogonal polarization. If the two wires were identical, had identical environments, and were excited equivalently, these two resonances would correspond to the symmetric (antisymmetric) low-frequency (highfrequency) mode of the coupled system of the two cut wires. However, in our case the symmetry is already broken by the excitation geometry and by the presence of the substrate. As a result, both resultant resonances have an antisymmetric character to some extent; thus both of them have a corresponding magnetic dipole moment connected to a resonance in magnetic permeability $\mu$. A comparison of Figs. 2(b) and $2(\mathrm{~d})$ shows the dependence of the resonance positions on $\mathrm{MgF}_{2}$ spacer thickness $d$. As expected from the description above of two coupled oscillators, the split between the two effective resonances depends on their coupling: For thin (thick) spacers, the coupling is strong (weaker); hence the two resonances are split by a large (smaller) amount in the spectrum.

The obvious polarization dependence of the cutwire pairs is undesirable, for example, in potential applications as perfect lenses. Thus we have also investigated samples for which the wire width equals the wire length, i.e., $w=l$. In this case the cut-wire pairs turn into nanoscopic plate pairs. Their measured optical properties are shown in Fig. 3. Obviously the resultant optical resonances are qualitatively similar, yet even more pronounced than for cutwire pairs. Reducing the value of $w=l$ allows for tuning of the resultant resonance positions.

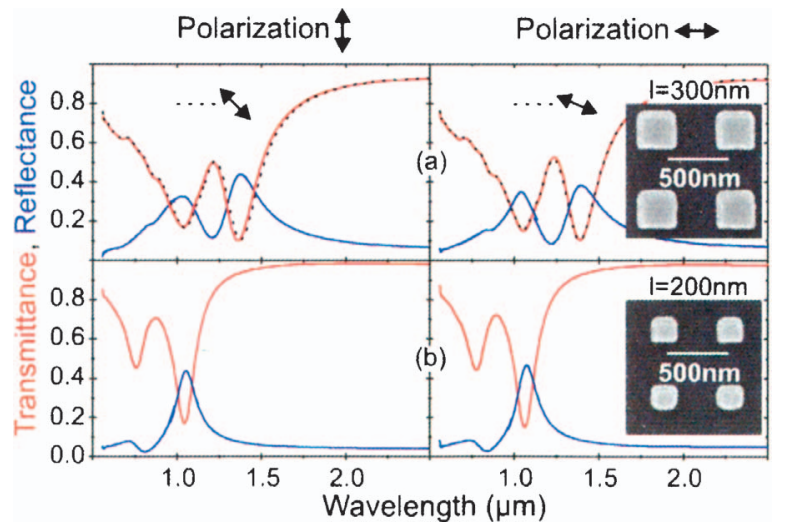

Fig. 3. Measured transmittance (red) and reflectance (blue) spectra for arrays of plate pairs. (a) $w=l=300 \mathrm{~nm}$, (b) $w=l=200 \mathrm{~nm}$. Fixed parameters: $t=20 \mathrm{~nm}, d=80 \mathrm{~nm}$, and $a_{x}=a_{y}=l+350 \mathrm{~nm}$. Orientation as in Fig. 2. Dotted curves, transmittance spectra for $45^{\circ}$ (left column) and $22.5^{\circ}$ (right column) incident polarization. 

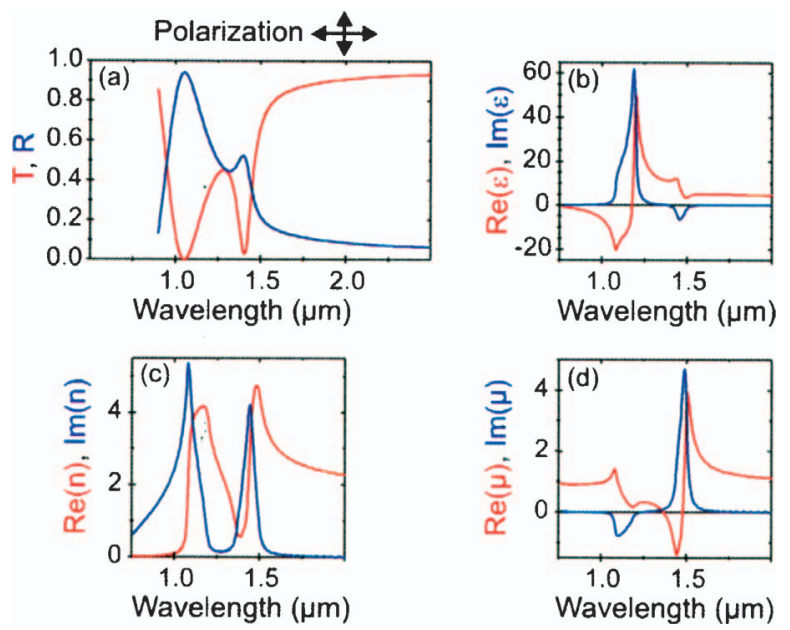

Fig. 4. (a) Calculated transmittance $T$ (red) and reflectance $R$ (blue) spectra for the structure shown in Fig. 3(a), i.e., for a single layer. Vertical and horizontal incident linear polarizations are strictly identical. (b) Electric permittivity $\epsilon$, (c) refractive index $n$, (d) magnetic permeability $\mu$ as obtained from the corresponding retrieval for a stack of layers.

For the reasons mentioned, the samples that correspond to Fig. 3(a) seem most attractive to us. Thus we explicitly present the retrieval of magnetic permeability $\mu$, electric permittivity $\epsilon$, and refractive index $n$ here. We use the Drude model for the gold ${ }^{4}$ and all other parameters as described above. The retrieval is done along the traditional lines ${ }^{14,15}$ : We calculate transmittance and reflectance spectra for a sequence of layers perpendicular to the substrate, i.e., for a periodic medium with lattice constant $a_{z}=130 \mathrm{~nm}$, and retrieve $\mu, \epsilon$, and $n$ from them. Figure 4 shows the calculated transmittance and reflectance spectra as well as the retrieval for the parameters of the structure shown in Fig. 3(a). Without any fitting parameters, the spectral positions of the resonances agree well. There are, however, quantitative deviations in the shape and the size of the resonances, which result in part from angle averaging in the experiment owing to the large N.A. For the long-wavelength resonance we indeed obtain a spectral region with a negative real part of $\mu$ but no negative $\operatorname{Re}(\epsilon)$ at the same frequency and no negative $\operatorname{Re}(n)$ (red curves). The corresponding imaginary parts of $\mu, \epsilon$, and $n$ (blue curves) are also depicted. The retrieved values for the short-wavelength resonance and beyond should be taken with some caution: As one gradually leaves the metamaterial limit, e.g., periodicity effects come into play. The retrieval for parameters that correspond to Fig. 3(b) is qualitative closely similar (also true for the cut-wire pairs of Fig. 2). In particular, we obtain $\operatorname{Re}(\mu)=-1.2$ at $1.0 \mu \mathrm{m}$ wavelength (not shown).

In conclusion, we have experimentally obtained and characterized metamaterials based on nanoscale cut-wire pairs and plate pairs. Comparison with theory and subsequent retrieval shows that both op- tions exhibit a frequency range with negative magnetic permeability. In contrast to other reports, ${ }^{16}$ however, we do not obtain a negative refractive index from the cut-wire pairs or plate pairs alone that we have studied so far.

We acknowledge support by the Deutsche Forschungsgemeinschaft through subproject A1.5 of the DFG-Forschungszentrum "Functional Nanostructures" and by project We1497/9-1. The research of C. M. Soukoulis is further supported by Alexander von Humboldt Senior-Scientist Award 2002, by the Ames Laboratory (contract W-7405-Eng-82), by European Union Future and Emerging Technologies project DALHM, and by the Defense Advanced Research Projects Agency (grant HR0011-05-C-0068). C. Enkrich's e-mail address is christian.enkrich@physik.uni-karlsruhe.de.

*Also with the Institute of Electronic Structure and Laser, Foundation for Research and Technology Helas, Heraklion, Crete, Greece.

\section{References}

1. R. A. Shelby, D. R. Smith, and S. Schultz, Science 292, 77 (2001).

2. T. J. Yen, W. J. Padilla, N. Fang, D. C. Vier, D. R. Smith, J. B. Pendry, D. N. Basov, and X. Zhang, Science 303, 1494 (2004).

3. D. R. Smith, J. B. Pendry, and M. C. K. Wiltshire, Science 305, 788 (2004).

4. S. Linden, C. Enkrich, M. Wegener, J. Zhou, T. Koschny, and C. M. Soukoulis, Science 306, 1351 (2004).

5. S. Zhang, W. Fan, B. K. Minhas, A. Frauenglass, K. J. Malloy, and S. R. J. Brueck, Phys. Rev. Lett. 94, 37402 (2005).

6. H. O. Moser, B. D. F. Casse, O. Wilhelmi, and B. T. Saw, Phys. Rev. Lett. 94, 63901 (2005).

7. N. Katsarakis, G. Konstantinidis, A. Kostopoulos, R. S. Penciu, T. F. Gundogdu, M. Kafesaki, E. N. Economou, Th. Koschny, and C. M. Soukoulis, Opt. Lett. 30, 1348 (2005).

8. V. G. Veselago, Sov. Phys. Usp. 10, 509 (1968).

9. J. B. Pendry, Phys. Rev. Lett. 85, 3966 (2000).

10. J. B. Pendry, A. J. Holden, D. J. Robbins, and W. J. Stewart, IEEE Trans. Microwave Theory Tech. 47, 2075 (1999).

11. A. N. Lagarkov and A. K. Sarychev, Phys. Rev. B 53, 6318 (1996).

12. L. V. Panina, A. N. Grigorenko, and D. P. Makhnovskiy, Phys. Rev. B 66, 155411 (2002).

13. V. A. Podolskiy, A. K. Sarychev, E. E. Narimanov, and V. M. Shalaev, J. Opt. A, Pure Appl. Opt. 7, 32 (2005).

14. D. R. Smith, S. Schultz, P. Markos, and C. M. Soukoulis, Phys. Rev. B 65, 195104 (2002).

15. Th. Koschny, P. Markos, E. N. Economou, D. R. Smith, D. C. Vier, and C. M. Soukoulis, Phys. Rev. B 71, 245105 (2005).

16. While completing this manuscript we learned about independent and related work on cut-wire pairs: V. M. Shalaev, in International Conference on Quantum Electronics and Laser Science (QELS) (Optical Society of America, 2005), paper JThA1. 